\title{
InN island shape and its dependence on growth condition of molecular-beam epitaxy
}

\author{
Y. G. Cao, ${ }^{\text {a) }}$ M. H. Xie, ${ }^{\text {b) }}$ Y. Liu, Y. F. Ng, and H. S. Wu \\ Department of Physics and HKU-CAS Joint Laboratory on New Materials, The University of Hong Kong, \\ Pokfulam Road, Hong Kong, China \\ S. Y. Tong \\ Department of Physics and Materials Science, City University of Hong Kong, Tat Chee Avenue, \\ Kowloon, Hong Kong
}

(Received 11 August 2003; accepted 27 October 2003)

\begin{abstract}
During molecular-beam epitaxy of InN films on $\mathrm{GaN}(0001)$ surface, three-dimensional (3D) islands are observed following an initial wetting layer formation. Depending on deposition condition, the 3D islands take different shapes. Pyramidal islands form when excess nitrogen fluxes are used, whereas pillar-shaped islands are obtained when excess indium fluxes are employed. The pillar-shaped islands are identified to represent the equilibrium shape, whereas the pyramidal ones are limited by kinetics. As the size of islands increases, their aspect ratio shows a decreasing trend, which is attributed to a gradual relaxation of strain in the layer by defects. (C) 2003 American Institute of Physics. [DOI: 10.1063/1.1635077]
\end{abstract}

III-V nitrides represent a family of important material system, which hold great promises in high temperature electronic and short wavelength optoelectronic applications. ${ }^{1,2}$ Heterostructures incorporating InGaN and $\mathrm{GaN}$ epilayers are essential ingredients in modern devices such as laser diodes and high-speed transistors. Employing InGaN quantum dots (QDs) embedded in GaN have been suggested a promising method to further improve the performance of such devices. ${ }^{3}$

As for many other heteroepitaxial systems, InGaN QDs are readily fabricated by self-assembly due to lattice mismatch with $\mathrm{GaN}^{4}$ This is the result of the StranskiKrastanow growth mode. ${ }^{5}$ Depending on the amount of strain, three-dimensional (3D) islands can be either coherent to the lattices of the substrate or defected, in which case, some of the strain is relieved by dislocations. ${ }^{6}$ The primary driving force for $3 \mathrm{D}$ islanding of the deposit during heteroepitaxy is to effectively relieve the strain, thereby the total energy of the system is minimized. ${ }^{6,7}$

In order to utilize self-assembled 3D islands as the QDs for device purposes, it is important to know the size and shape of the islands, as these factors determine electric/ optical properties of the structure. For island shape, arguments based on thermodynamic and kinetic considerations have all been put forward. ${ }^{8-11}$ However, it remains an open question whether the islands take the equilibrium shape or are kinetically limited. It is thus of great scientific interests, in addition to being practically relevant, to investigate the shape of 3D islands.

In this letter, 3D InN island shape and its dependence on flux conditions of molecular-beam epitaxy (MBE) are followed. By simultaneously monitoring the lattice spacing using reflection high-energy electron diffraction (RHEED), it

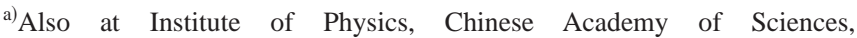
Beijing 100080, China.

b) Author to whom correspondence should be addressed; electronic mail: mhxie@hkusua.hku.hk
}

was concluded that the islands were dislocated and the (residual) strain in an island depended on its size. ${ }^{4}$ This is in contrast to the case of coherent islands where no defect is present and strain accommodation is by lattice distortion (elastic relaxation). This letter shows that such a difference leads to opposite trends in island aspect ratio as a function of island size. It is also found that the shape of islands depends on the fluxes employed during MBE. Pyramidal islands are observed when excess nitrogen $(\mathrm{N})$ fluxes are used, whereas pillar-shaped islands are obtained when excess indium (In) fluxes are adopted. The pillar-shaped islands having low aspect ratios represent the equilibrium shape, whereas pyramidal islands with higher aspect ratios are limited by kinetics during MBE growth.

Film growth and surface examination were conducted in a multichamber ultrahigh vacuum system, the details of which have been described elsewhere. ${ }^{4,12}$ Briefly, the system constituted, among others, a MBE reactor and a scanning tunneling microscope (STM). In MBE, conventional Knudsen cells for gallium $(\mathrm{Ga})$, indium, and a radio-frequency (rf) plasma unit for $\mathrm{N}$ were installed together with the RHEED facility. Prior to $\mathrm{InN}$ deposition, a $\mathrm{GaN}$ buffer film $(>1 \mu \mathrm{m}$ thick) was grown on $\mathrm{SiC}(0001)$ substrate under the Ga-rich flux condition. Afterwards, the sample was briefly annealed to desorb excess $\mathrm{Ga}$ atoms from the surface before being cooled down to $370{ }^{\circ} \mathrm{C}$. Subsequent $\mathrm{InN}$ deposition was carried out under various In fluxes, while the flux of $\mathrm{N}$ was kept constant at the growth rate equivalence of 0.033 bilayers per second (BLs/s). After some specified coverage, the growth was stopped and the sample quenched rapidly for subsequent STM measurements in an adjacent chamber. All the STM experiments were conducted at room temperature under the constant current mode. The tunneling current was $0.1 \mathrm{nA}$ and the sample bias was $-2.0 \mathrm{~V}$.

Figures 1(a) and 1(b) show typical surfaces following 4.4 BLs InN deposition under the In fluxes of 0.009 and $0.056 \mathrm{BL} / \mathrm{s}$, or with the In/ $\mathrm{N}$ flux ratios of 0.3 and 1.7 , re- 

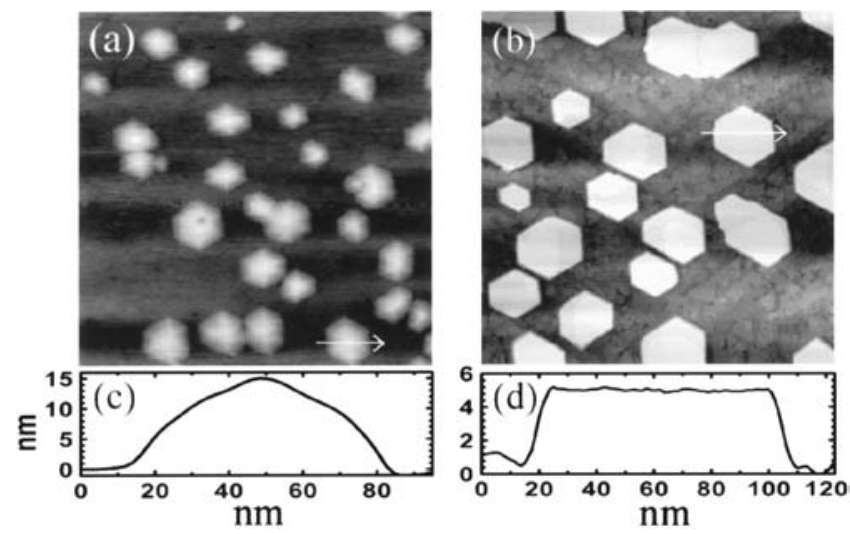

FIG. 1. STM images depicting the shape of 3D InN islands grown on $\mathrm{GaN}(0001)$ using (a) excess-N and (b) excess-In fluxes. Line profiles across typical islands are shown in (c) and (d), respectively. Image size: $500 \mathrm{~nm}$ $\times 500 \mathrm{~nm}$.

spectively. The film growth rate was also changed accordingly from 0.009 to $0.033 \mathrm{BLs} / \mathrm{s}$, where the former was determined by In flux while the latter was limited by $\mathrm{N}$ flux. From the images, it is seen that $3 \mathrm{D}$ islands have formed in both cases on otherwise flat surfaces. As noted earlier, the 3D islands are partially strained with defects inside. ${ }^{4}$ Comparing Figs. 1(a) and 1(b), an apparent difference regarding island shape is noted. In Fig. 1(a), the islands are pyramidal with the sidewalls being composed of stacks of double bilayer steps. ${ }^{13}$ On the other hand, for islands in Fig. 1(b), they are pillars with flat tops. Such a difference is better revealed by the line profiles given in Figs. 1(c) and 1(d). Experiments over a range of fluxes suggest that the pillar-shaped islands are characteristic to growth using excess-In fluxes (i.e., $\mathrm{In} / \mathrm{N}>1)$, whereas pyramidal islands form only under excess- $\mathrm{N}(\mathrm{In} / \mathrm{N}<1)$ conditions.

Figures 2 and 3 compile, respectively, the height and aspect ratio of islands for cases where $\mathrm{In} / \mathrm{N}$ flux ratios are 0.3 and 1.7. All data are obtained from STM measurements. From the plots, general trends are noted where the height increases whereas the aspect ratio decreases with increasing island size. However, the data corresponding to low $\mathrm{In} / \mathrm{N}$ flux ratio are very scattered. For islands corresponding to $\mathrm{In} / \mathrm{N}$ $\sim 1.7$, the data are more consistent. In addition, islands formed under high $\mathrm{In} / \mathrm{N}$ ratios are generally low in height, so

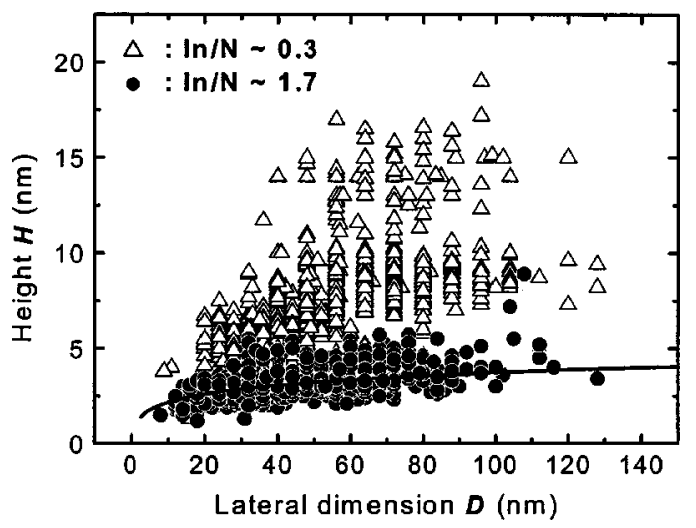

FIG. 2. Island heights plotted as a function of lateral size for those grown under In/N flux ratios of 0.3 (open triangles) and 1.7 (filled circles) during MBE. The solid line is a theoretical curve taking account the gradual relaxation of strain in islands. edge barrier is diminished and interlayer diffusion of atoms
Downloaded 07 Nov 2006 to 147.8.21.97. Redistribution subject to AlP license or copyright, see http://apl.aip.org/apl/copyright.jsp

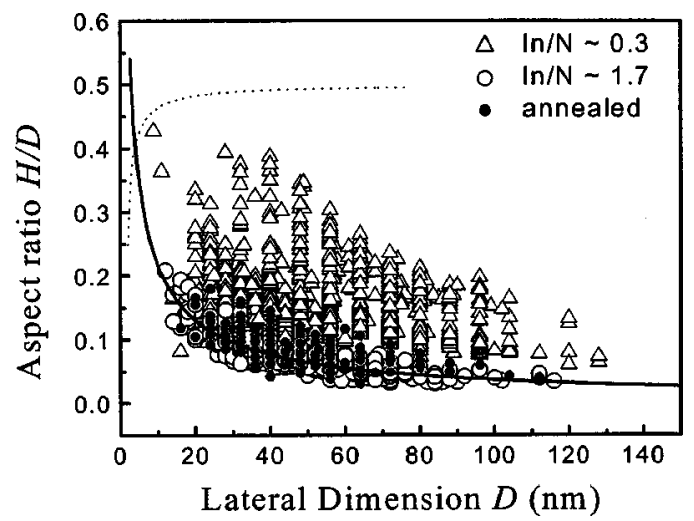

FIG. 3. Aspect ratio of islands plotted against lateral size for growth under $\mathrm{In} / \mathrm{N}$ flux ratios of 0.3 (open triangles) and 1.7 (open circles). Data from annealed samples are shown by filled circles. The dotted line is a theoretical curve, assuming the islands are coherent, whereas the solid curve is obtained taking into account partially relaxation of strain by defects.

are the aspect ratios. In Fig. 3, results from two annealed samples are also presented, which reveal further difference between the two types of islands. Little change is observed for islands grown under $\mathrm{In} / \mathrm{N}>1$, whereas significant reduction in island aspect ratio is observed for those initially formed under $\operatorname{In} / \mathrm{N}<1$. Here, the annealing time was $2 \mathrm{~min}$ and the annealing temperature was $370^{\circ} \mathrm{C}$, the same as that for deposition. During annealing, the In flux was shut off, while the flow rate of $\mathrm{N}_{2}$ gas was maintained to the value used for deposition but the rf power to the plasma unit was turned off, so there was no reactive $\mathrm{N}$ species present.

The first conclusion can be drawn from the above results is that $\mathrm{InN}$ islands formed during deposition under excess-In fluxes are close to equilibrium, as otherwise, annealing of the sample would result in a change, as did for those formed under excess-N condition. For the latter, island growth is kinetically limited, which is also consistent with the scattering of the data.

The question is why there is such a dependence of island shape on flux condition of MBE. We speculate it is related to a change of surface condition under two different growth regimes. Recent experiments have suggested that under excess-In condition, the surface is covered by metallic In layers, which act as surfactant affecting both surface energetics and kinetic barriers of the system. ${ }^{14}$ Indium surfactant can enhance adatom diffusion on surface, thereby, helps the system to reach its equilibrium. On the other hand, for growth using excess- $\mathrm{N}$ fluxes, full layer of In atoms does not exist and therefore, it leads to slow kinetics and consequently islands away from equilibrium.

The even higher aspect ratio for the kinetics-limited islands than equilibrium ones implies a kinetic process which gives rise to additional uphill current than that due to strain itself. As is known, if there exists an energy barrier for adatoms hopping down a step (Ehrlich-Schwoebel barrier), an uphill current will be created. ${ }^{15,16}$ In nitride growth, analyses of island distributions across terraces provide such a hint for surfaces without excess metallic layers, ${ }^{17}$ which precisely corresponds to growth under excess- $\mathrm{N}$ conditions. On the other hand, for surfaces with excess metallic layers, the step 
readily occurs and equilibrium shape of islands is quickly achieved.

Another observation is the decreasing trend of island aspect ratio, as given in Fig. 3. Such a trend contradicts to that of 3D InAs and Ge islands, where an increasing trend has been noted. ${ }^{18,19}$ Theoretical investigations have provided explanations to such increasing trend, ${ }^{8,10,11}$ which lies in the enhancement of strain relaxation in tall islands. For dislocated islands such as that of $\mathrm{InN}$ on $\mathrm{GaN}$, the (residual) strain inside an island depends on its size, as additional dislocations can be continuously introduced as the islands grow. Consequently, the driving force for vertical growth of the film becomes weaker.

To put it quantitatively, we adopt the approach of Tersoff and Tromp, ${ }^{11}$ where the total energy of the system is considered. It was shown that for a system with lattice-misfit strain $f$, system energy would be minimized when the size of a coherent $3 \mathrm{D}$ island was given by ${ }^{11}$

$$
D=e \phi H e^{\Gamma / c H},
$$

where $D$ is the lateral size of the 3D island and $H$ is its height. Constant $\Gamma$ represents the surface tension of the film, and $\phi=e^{-3 / 2} \cot \theta, \theta$ is the contact angle between the island and the wetting layer. $c=\sigma^{2}(1-\nu) / 2 \pi G$ is associated with the stress in film, $\sigma \sim 2 G f(1+\nu) /(1-\nu)$, and $\nu, G$ are Poisson ratio and shear modules of the material, respectively. Based on the above, one may calculate the (equilibrium) aspect ratio of islands and the resulting trend is depicted by the dotted line in Fig. 3. The increasing trend agrees with the experiments for coherent islands as mentioned earlier. For dislocated islands, the stress in film is no longer constant, but evolves as island grows. For example, for a continuum layer with thickness $H$ greater than the critical thickness for dislocation formation, the residual strain, $\epsilon$, in film becomes ${ }^{20}$

$$
\epsilon \sim \frac{b}{4 \pi(1+\nu) H} \ln \left(\frac{4 H}{b}\right),
$$

when the strain-relieving dislocations are of edge type having the Burgers vector $b$. Assuming the same for 3D islands, we estimate the strain in islands according to Eq. (2) and then put it in place of $f$ in Eq. (1). The calculated result is shown by the solid curves in Figs. 2 and 3 using the parameters: $b=0.35 \mathrm{~nm}, \nu=1 / 3$, and $\Gamma / G=6.0 \times 10^{-9} \mathrm{~cm}^{-1}$. It is seen that it describes well the experimental data, especially for islands grown under excess-In conditions, which are known to be at equilibrium.

To summarize, pyramidal and pillar-shaped islands of InN are observed on $\mathrm{GaN}(0001)$ following growth by MBE. The pillar-shaped islands having low aspect ratios are close to equilibrium, which are formed only when excess-In fluxes are used during deposition. Formation of pyramidal islands under excess-N fluxes is kinetically limited. The decreasing trend of island aspect ratio with respect to island size is attributed to gradual relaxation of residual strain in dislocated islands.

The authors would like to acknowledge the technical support from W. K. Ho. This work is supported by grants from the Research Grants Council of the Hong Kong Special Administrative Region, China (project Nos. HKU7096/01P and HKU7118/02P).

\footnotetext{
${ }^{1}$ S. Nakamura, IEEE Circuits Devices Mag. 11, 19 (1995).

${ }^{2}$ S. Strite and H. Morkoç, J. Vac. Sci. Technol. B 10, 1237 (1992).

${ }^{3}$ D. Bimberg, M. Grundmann, and N. N. Ledentsov, Quantum Dot Heterostructures (Wiley, London, 1999).

${ }^{4}$ Y. F. Ng, Y. G. Cao, M. H. Xie, X. L. Wang, and S. Y. Tong, Appl. Phys. Lett. 81, 3960 (2002).

${ }^{5}$ E. Bauer and J. H. van der Merwe, Phys. Rev. B 33, 3657 (1986).

${ }^{6}$ C. Ratsch and A. Zangwill, Surf. Sci. 293, 123 (1993).

${ }^{7}$ J. Tersoff and F. K. LeGoues, Phys. Rev. Lett. 72, 3570 (1994).

${ }^{8}$ L. G. Wang, P. Kratzer, M. Scheffler, and N. Moll, Phys. Rev. Lett. 82, 4042 (1999); L. G. Wang, P. Kratzer, N. Moll, and M. Scheffler, Phys. Rev. B 62, 1897 (2000).

${ }^{9}$ N. Moll, M. Scheffler, and E. Pehlke, Phys. Rev. B 58, 4566 (1998).

${ }^{10}$ I. Daruka, J. Tersoff, and A.-L. Barabási, Phys. Rev. Lett. 82, 2753 (1999).

${ }^{11}$ J. Tersoff and R. M. Tromp, Phys. Rev. Lett. 70, 2782 (1993).

${ }^{12}$ S. M. Sean, M. H. Xie, W. K. Zhu, L. X. Zheng, H. S. Wu, and S. Y. Tong, Surf. Sci. 445, L71 (2000).

${ }^{13}$ M. H. Xie, S. M. Seutter, W. K. Zhu, L. X. Zheng, H. S. Wu, and S. Y. Tong, Phys. Rev. Lett. 82, 2749 (1999).

${ }^{14}$ C. Kruse, S. Einfeldt, T. Böttcher, and D. Hommel, Appl. Phys. Lett. 79, 3425 (2001)

${ }^{15}$ M. D. Johnson, C. Orme, A. W. Hunt, D. Graff, J. Sudijono, L. M. Sander, and B. G. Orr, Phys. Rev. Lett. 72, 116 (1994).

${ }^{16}$ M. F. Gyure, J. J. Zinck, C. Ratsch, and D. D. Vvedensky, Phys. Rev. Lett. 81, 4931 (1998).

${ }^{17}$ M. H. Xie, L. X. Zheng, and S. Y. Tong (unpublished).

${ }^{18}$ H. Saito, K. Nishi, and S. Sugou, Appl. Phys. Lett. 74, 1224 (1999).

${ }^{19}$ G. Medeiros-Ribeiro, A. M. Bratkovski, T. I. Kamins, D. A. A. Ohlberg, and R. S. Williams, Science 279, 353 (1998).

${ }^{20}$ J. Y. Tsao, Materials Fundamentals of Molecular Beam Epitaxy (Academic, San Diego, 1993).
} 